\section{Radiofrequenztherapie bessert Fersenschmerz}

\author{
Bei plantarem Fersenschmerz werden viele Methoden empfohlen. Doch \\ evidenzbasierte Daten sind rar. Forscher aus Shanghai sind das Problem jetzt \\ erneut angegangen - scheinbar mit Erfolg.
}

P lant antarer Fersenschmerz ist kein seltenes Phänomen, die Prävalenzraten reichen von 3,6 bis 7,5\%. Für die Wirksamkeit verschiedener Maßnahmen wie Einlagen, lokale Kortikoidtherapien oder Stretching des M. gastrocnemius zur Verminderung von Kontrakturen gibt es nur geringe Evidenz.

Im Rahmen einer randomisierten, kontrollierten Studie wurde nun versucht, bei 100 Patienten durch Inaktivierung der Triggerpunkte des $M$. gastrocnemius und Entlastung des Wadenmuskels eine Befreiung vom Fersenschmerz zu erzielen. Bei einem Teil der Patienten wurde der M. gastrocnemius mit ultraschallgeführter gepulster $\mathrm{Ra}$ diofrequenztherapie (UG-PRF) behandelt, die Probanden der Kontrollgruppe erhielten eine Scheinbehandlung.

Die Beschwerden wurden anhand des Foot Health Status Questionnaire (FHSQ-Pain) und der visuellen Analogskala (VAS-“first-step“ pain) beurteilt.
Nach Lokalisation des Triggerpunkts wurden zur Lokalanästhesie $3 \mathrm{ml}$ Levobupivacain $(0,5 \%)$ injiziert. Die PRF unter Ultraschallkontrolle erfolgte bei $42^{\circ} \mathrm{C}$ für fünf Minuten einmal pro Woche über insgesamt drei Wochen. Lagen mehrere Triggerpunkte vor, wurde die Behandlung wiederholt. In der Kontrollgruppe erfolgten die gleichen Schritte, bis auf die Zufuhr der Radiofrequenzenergie.

Drei Monate nach UG-PRF-Therapie hatten so Behandelte im FHSQ-painScore (0 bis 100 Punkte) im Vergleich zu Patienten mit Scheinbehandlung 20 Score-Punkte weniger, nach sechs Monaten 18 Punkte weniger. Dies entspricht einer Schmerzreduktion im Vergleich zum Ausgangswert um $87 \%$ (versus $47 \%$ in der Kontrollgruppe) nach drei Monaten und um $71 \%$ (versus 34,5\% in der Kontrollgruppe) nach sechs Monaten. Die Werte auf der VAS-"first-step" pain hatten sich im Vergleich zur Kontrollgruppe um 26,1 $\mathrm{mm}$ nach drei Monaten und 14,3 mm nach sechs Monaten verbessert. Dies entspricht einer Schmerzreduktion von 48 versus $15 \%$ sowie 40 und $23 \%$.

Auch die Funktion des Fußes sowie die allgemeine Fußgesundheit hatten sich nach drei Monaten im Vergleich zur Scheinbehandlungsgruppe gebessert (FHSQ-foot function 42 versus 6\%; FHSQ-general foot health 51 versus $26 \%$ ), ebenso die körperliche Funktionsfähigkeit nach drei und sechs Monaten im SF-36 (bei UG-PRF 11 beziehungsweise 10 Punkte mehr als in der Kontrollgruppe).

Auf der psychischen Ebene ergaben sich dagegen keine Unterschiede. Insgesamt wurden keinerlei Komplikationen beobachtet.

Fazit: Die UG-PRF-Therapie bei plantarem Fersenschmerz scheint sowohl effektiv als auch sicher zu sein und ist damit eventuell eine therapeutische Alternative.

Dr. Christine Starostzik

Ye Le et al. A Comparative Efficacy Evaluation of Ultrasound-Guided Pulsed Radiofrequency Treatment in the Gastrocnemius in Managing Plantar Heel Pain: A Randomized and Controlled Trial. Pain Medicine 2015; 16 (4): 782-90.

\section{Frühe Bildgebung bei älteren Patienten mit akuten Rückenschmerzen nutzlos}

\begin{abstract}
Ältere Patienten mit neu aufgetretenen Kreuzschmerzen haben langfristig nicht weniger Beschwerden, wenn sie schon vor Ablauf von sechs Wochen einer bildgebenden Untersuchung unterzogen werden. Auch bedrohliche Ursachen werden offenbar nicht häufiger entdeckt.
\end{abstract}

B ei akuten Kreuzschmerzen wird in der Regel keine bildgebende Diagnostik empfohlen - es sei denn, es liegen Warnhinweise für gefährliche Verläufe vor. Zu diesen „red flags“ gehört laut der Nationalen Versorgungsleitlinie Kreuzschmerz auch höheres Alter der Betroffenen, weil es mit einem erhöhten Risiko für Tumorleiden einhergeht. In der entsprechenden europäischen Leitlinie wird schon ein Alter ab 55 Jahren als Warnhinweis genannt, der eine frühe Bildgebung rechtfertigen kann.
Daten einer jetzt veröffentlichten Studie stellen diese Empfehlungen infrage. An der prospektiven Studie waren 5.145 Ältere ( $\geq 65$ Jahre) beteiligt, die sich ihrem Hausarzt wegen neu aufgetretener Kreuzschmerzen vorgestellt hatten. Innerhalb der ersten sechs Wochen waren bei $26 \%$ eine konventionelle Röntgenuntersuchung und bei 7,5\% eine Computer(CT)- oder Magnetresonanztomografie (MRT) angefertigt worden.

1.174 geröntgte Patienten und $349 \mathrm{~Pa}$ tienten mit CT oder MRT wurden je- weils mit ebenso vielen Patienten ohne frühe Bildgebung verglichen. Das Ergebnis: Die rückenschmerzbedingten körperlichen Einschränkungen, erfasst mit dem Roland-Morris Disability Questionnaire (RMDQ), waren nach drei, sechs und zwölf Monaten jeweils mit und ohne Bildgebung identisch. Auch im Hinblick auf Schmerzen und Lebensqualität ergaben sich keine klinisch relevanten Unterschiede.

Fazit: Der einzige Unterschied zwischen den Gruppen mit und ohne frühe Bildgebung zeigte sich auf der Kostenseite. Die Gesamtkosten lagen in der Röntgengruppe um $27 \%$, in der MRT-/CT-Gruppe um $30 \%$ höher. Dr. Beate Schumacher

Jarvik JG et al. Association of early imaging for back pain with clinical outcomes in older adults. JAMA. 2015;313(11):1143-53. 